\title{
Ziel: Sepsis früh erkennen und behandeln
}

Sepsis ist die Hauptursache für Todesfälle im Krankenhaus, für Wiederaufnahmen im Spital und für hohe Gesundheitskosten. Sie ist damit eines der weltweit größten Gesundheitsprobleme. Anlässlich des WeltSepsis-Tages Mitte September betonte die Österreichische Gesellschaft für Anästhesiologie, Reanimation und Intensivmedizin (ÖGARI) einmal mehr die Bedeutung von Früherkennung und geeigneten Rahmenbedingungen für ein optimales Sepsis-Management.

„In den mehr als eineinhalb Jahren COVID 19-Pandemie sind mehr Menschen denn je auf kritische Infektionserkrankungen, die eine intensivmedizinische Therapie erforderlich machen, und auf kritische Krankheitszustände wie ein Multiorganversagen aufmerksam geworden", sagt ÖGARIPräsident Univ.-Prof. Dr. Walter Hasibeder, Leiter der Abteilung für Anästhesie und operative Intensivmedizin am Krankenhaus St. Vinzenz Zams.

\section{Jahre Welt-Sepsis-Tag}

"Seit 10 Jahren wird der Welt-SepsisTag begangen. Und in dieser Zeit sind auf gesundheitspolitischer Ebene wichtige Fortschritte gelungen, unter anderem eine WHO-Resolution, die alle Mitgliedsstaaten aufruft, effektive Maßnahmen gegen Sepsis in ihre nationalen Gesundheitsstrategien zu integrieren“, erklärt Hasibeder. „Um für Prävention, Früherkennung, Dia-

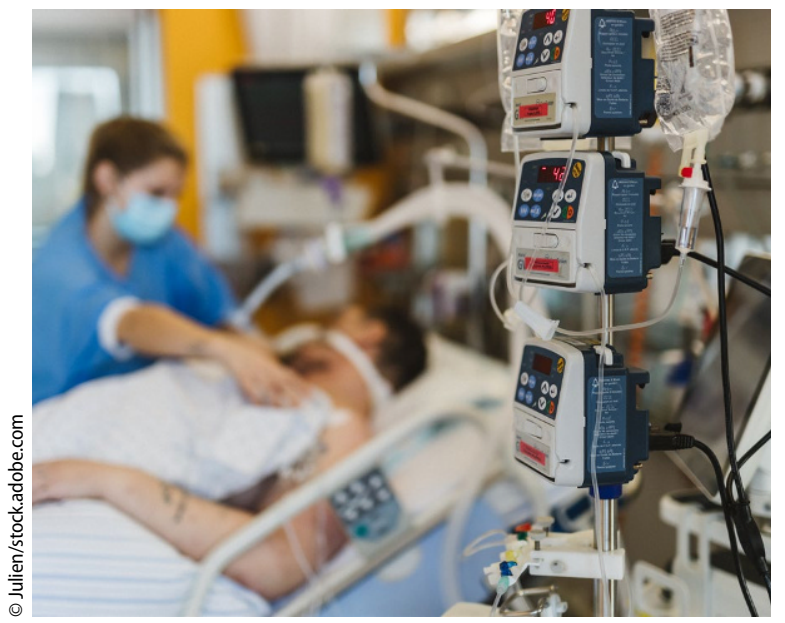

$\Delta$ Auch nach der Behandlung auf der Intensivstation bedürfen Patienten aufgrund dieses dramatischen Ereignisses besonderer Betreuung. gnose, Behandlung und langfristige Nachbetreuung in der Gesundheitsplanung in Österreich optimale Rahmenbedingungen zu definieren und entwickeln, erarbeiten Expertinnen und Experten nun gemeinsam mit der Gesundheitspolitik einen Nationalen Aktionsplan Sepsis (NAP-Sepsis)", so Assoc.-Prof. Priv.-Doz. Dr. Eva Schaden, von der ÖGARI und Leiterin einer Intensivstation an der Univ.-Klinik für Anästhesie, Allgemeine Intensivmedizin und Schmerztherapie an der MedUni Wien. Ein wichtiges Element sei dabei die Erhebung valider Daten über Häufigkeit und Sterblichkeitsrate der Sepsis in Österreich. „Wir müssen im Moment mit Hochrechnungen und Näherungswerten von internationalen Zahlen arbeiten, weil uns entsprechende Datengrundlagen auf nationaler Ebene fehlen." Auf Basis der Hochrechnung deutscher Zahlen ist in Österreich von etwa 28.000 Sepsis-Erkrankten und 6700 Sepsis-bedingten Todesfällen pro Jahr auszugehen. Gespräche mit dem Gesundheitsministerium sollen die Etablierung eines Intensivregisters in die Wege leiten. Damit will man über die Demographie der Patientinnen und Patienten auf den österreichischen Intensivstationen ebenso Aufschluss gewinnen wie über ihre Diagnosen, Komplikationen oder Therapien. „Solche Daten hätten uns seit dem Pandemiebeginn nützliche Informationen geliefert, und sie werden es auch in Bezug auf die Sepsis tun können", so Schaden.

\section{Post-Sepsis-Care}

Die Phänomene Post-COVID oder Long-COVID haben in den vergan- genen Monaten vielen Menschen bewusst gemacht, dass bei kritischen Erkrankungen mit dem Ende der intensivmedizinischen Behandlung der Versorgungsbedarf nicht abgeschlossen ist. Das Problem sei allerding nicht auf COVID-19 beschränkt, stellt Schaden fest. Bis zu $50 \%$ der Menschen, die eine Sepsis überstanden haben, leiden langfristig an körperlichen oder psychischen Folgen dieser schweren Erkrankung, die geeignete Betreuungsstrukturen und -einrichtungen erfordern. Die weiterführende Behandlung und Versorgung nach der Entlassung aus der Intensivstation - die "Post-ICU Care" - gewinne insgesamt zunehmend an Bedeutung.

Eine besondere Bedeutung kommt beim Sepsis-Management auch der Früherkennung zu, denn je später die Diagnosestellung und eine angemessene Therapie erfolgt, desto schlechter sind Heilungs- und Überlebenschancen und desto dramatischer ist der Verlauf. Aber $80 \%$ der Sepsis-Fälle treten außerhalb eines Krankenhauses auf. „Bewusstsein für Alarmsignale der Sepsis ist daher zentral", betont Hasibeder.

Hinweis des Verlags. Der Verlag bleibt in Hinblick auf geografische Zuordnungen und Gebietsbezeichnungen in veröffentlichten Karten und Institutsadressen neutral.

Wien klin Mag 2021 · 24:204-205 https://doi.org/10.1007/s00740021-00418-9

(C) Springer-Verlag GmbH Austria, ein Teil von Springer Nature 2021 
Hier steht eine Anzeige.

Springer 